\title{
Metformin and its gastrointestinal problems: A review.
}

\author{
Madiha Fatima $^{1}$, Saleha Sadeeqa ${ }^{1 *}$, Saeed Ur Rashid Nazir ${ }^{2}$ \\ ${ }^{1}$ Institute of Pharmacy, Lahore College for Women University, Lahore, Pakistan \\ ${ }^{2}$ Department of Pharmacy, University of Sargodha, Pakistan
}

\begin{abstract}
Metformin is a biguanide class of drugs and has been recommended as first-line therapy for type 2 diabetes. It has a good safety profile, efficacy, comparatively reduced cost, and potential cardiovascular benefits. Metformin is an insulin-sensitizing agent, its bioavailability is $50 \%-60 \%$. Generally, A1C levels are lowered by $1.5 \%$ points by metformin monotherapy. Treatment with metformin decreases fasting plasma glucose concentrations by $25 \%$ to $30 \%$ and decreases the production of glucose. Metformin reduces hepatic glucose production and absorption of glucose in the intestine. In addition to it, decreases fatty acids oxidation. In liver and skeletal muscles the mitochondrial function and AMP Activated Protein Kinase (AMPK) activity are considered as potential mechanisms and has gained much attention by which metformin exerts its advantageous effects. In the gut enteroendocrine cells secret glucagon-like peptide-1 and glucose-dependent insulinotropic peptide, which are considered as important determinants for the disposal of glucose following a meal. Glucose production is reduced either by decreasing gluconeogenesis or by glycogenolysis. Treatment with metformin is, nevertheless, very often associated with gastrointestinal side effects and quality of life and treatment adherence is negatively affected in patients of type 2 diabetes. The most common gastrointestinal symptoms are diarrhea, heartburn, and nausea, followed by abdominal pain, bloating, and retching. The mechanism lying under gastrointestinal intolerance caused by metformin is unclear. However, there are different hypothesis proposed, including stimulation of intestinal secretion of serotonin, alteration in incretin and metabolism of glucose, and malabsorption of bile salts. Metformin is used clinically in diabetes, polycystic ovary syndrome, and in obese for weight reduction. It has cardioprotective effect and its use is recently being studied in cancer and HIV associated metabolic abnormalities.
\end{abstract}

Keywords: Metformin, Diabetes, Gastrointestinal, Insulin, Glucose.

Accepted on April 17, 2018

\section{Introduction}

Metformin is the first-line antidiabetic agent, which is used for type 2 diabetes treatment, particularly in overweight patients where diabetes not controlled by adequate diet. It lies under the class of biguanides, which are guanidine derivative. Chemically it is 1,1-dimethylbiguanide hydrochloride. In the market it is the only biguanide derivative available. United States Food and Drug Administration (FDA) approved its use in 1994 [1]. In 1970s, the first two biguanides, buformin and phenformin were withdrawal from the US market due to higher risk of lactic acidosis [2].

\section{Metformin Pharmacokinetics}

Metformin is the accepted diabetes treatment and has a good safety profile [3]. It is an insulin-sensitizing agent [4]. Its bioavailability without binding to protein is approximately $50-60 \%$, and it has large volume of distribution and in small intestine it is maximum accumulated [5]. It has been found that $500 \mathrm{mgdose}$ of metformin has absolute bioavailability of $50 \%$ approximately and by increasing the dose bioavailability decreases [6]. In the presence of food, bioavailability decreases with an Area under the Curve (AUC) 20\% lower and maximum plasma concentration $\left(\mathrm{c}_{\max }\right) \quad 35 \%$ lower [7]. However, with regular doses, the steady-state plasma concentration reaches within 24-48 h having plasma half-life: 2.7-4 h [8]. 63 to $276 \mathrm{~L}$ is the range of volume of distribution. Metformin hydrochloride has renal clearance of $>400 \mathrm{ml} / \mathrm{min}$. It is eliminated via glomerular filtration and tubular secretion. In patients having impaired renal function, renal clearance decreases as the creatinine clearance decreases. This results in prolonged elimination half-life and raised plasma concentrations [1]. Generally, A1C levels are lowered by $1.5 \%$ points by metformin monotherapy $[9,10]$.

\section{Mechanism of Action}

Metformin reduces hepatic glucose production and absorption of glucose in the intestine. In addition it decreases fatty acids oxidation. Furthermore, it increases sensitivity of insulin, 
resulting in decrease resistance of insulin that is commonly a problem in type 2 Diabetes Mellitus (T2DM) patients [11]. Treatment with metformin decreases fasting plasma glucose concentrations by $25 \%$ to $30 \%$ and decreases the production of glucose [12]. Metformin for its access to hepatocytes utilizes the Organic Cation Transporter-1 (OCT-1). It is considered that in hepatocytes it alters mitochondrial function and activity of AMP Activated Protein Kinase (AMPK) [13], resulting in reduced hepatic production of glucose and decreasing glucose, while activation of AMPK in skeletal muscles may augment utilization of glucose [14].

In liver and skeletal muscles the mitochondrial function and AMPK activity have considered as potential mechanisms and has gained much attention by which metformin exerts its advantageous effects. Metformin improves the lipid profile, in polycystic ovarian syndrome, restores the function of ovaries, and decreases infiltration of fats in the liver and lesser microvascular and macrovascular complications related to T2DM. Recently, in studies metformin has been found as an advent treatment for cancer, as gestational diabetes treatment restores function of ovaries and in pre-diabetic individuals for preventing T2DM [15].

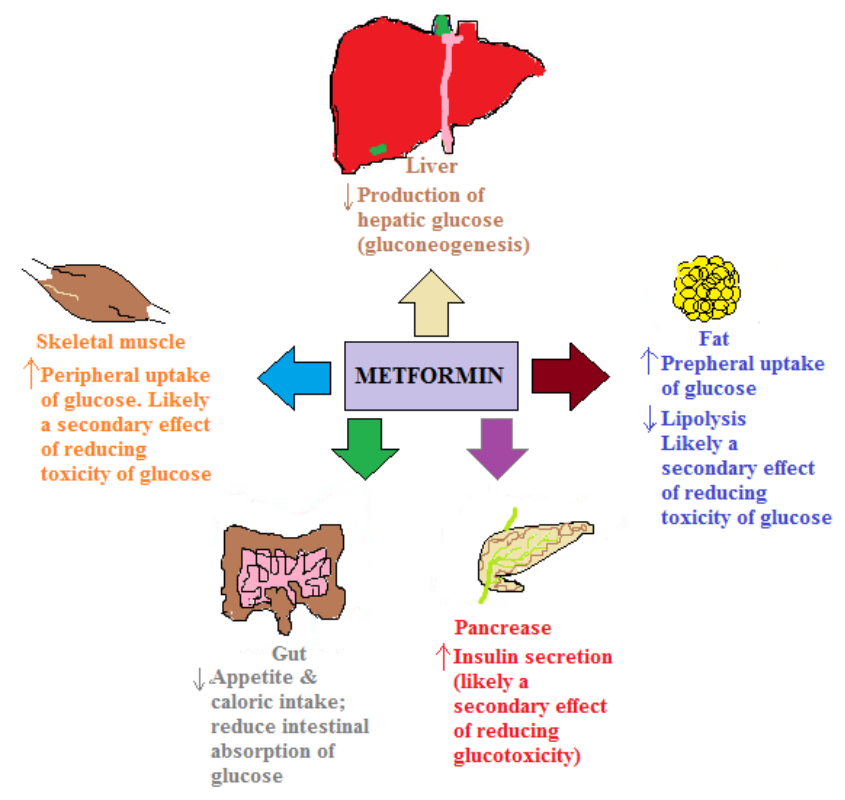

Figure 1. Mechanism of action of metformin.

In the gut, enteroendocrine cells secrete Glucagon-Like Peptide-1 (GLP-1) and Glucose-Dependent Insulinotropic Peptide (GIP), which are considered as important determinants for the disposal of glucose following a meal [16]. Glucose production is reduced either by decreasing gluconeogenesis [17] or by glycogenolysis [18,19]. Although in assessing the rates of these metabolic processes there are limitations in these studies in terms of methodologies used [12]. To a lesser extent, the glucose levels in the plasma are lowered by increasing peripheral glucose uptake by the adipose tissues and skeletal muscles. Most likely it is secondary to the glucotoxicity reversal and is not a direct pharmacological effect [20]. The trafficking of glucose transporters 1 and 4 are facilitated to the plasma membrane by metformin in the skeletal muscles and adipose tissues in the presence of insulin [21]. Metformin in the small intestine is found in higher concentrations. It may also decrease absorption of glucose in the intestine thereby, influencing postprandial hyperglycaemia [5].

Mechanism of action of metformin is depicted in Figure 1.The upward arrows show an increase and downward arrows show a decrease.

\section{Metformin Clinical Uses}

\section{Diabetes}

For type 2 diabetes body mass index has been found to be a major risk factor. There is $5 \%$ increase in risk of diabetes for every $1 \mathrm{~kg}$ gain in body weight. Metformin is an effective therapy in reducing weight and preventing diabetes. Metformin therapy is a reasonable options for overweight mild hyperglycaemic individuals particularly those who failed in life style modification.

\section{Polycystic ovary syndrome}

In patients with PCOS metformin improve the metabolic and reproductive abnormalities. At dosages of $1500 \mathrm{mg} / \mathrm{d}$, metformin decreases; body weight, insulin, testosterone and luteinizing hormone levels and 17-hydroxy progesterone response to human chorionic gonadotropin. Metformin has also been found to be effective in inducing ovulation than clomiphene. Metformin lies under category B drug in pregnancy and is used in women with gestational diabetes. Metformin in PCOS patients increases the chances of conception and preventing early trimester pregnancy loss.

\section{Weight reduction}

Metformin is used as a tool in non-diabetic obese patients to reduce weight. Many studies were conducted and concluded that metformin increases weight loss [5].

\section{Cardiovascular protection}

Metformin in addition to its anti-hyperglycemic properties might have cardiovascular protective actions. Metformin therapy exerts its cardioprotective effects by; decreasing hyperglycemia, total cholesterol levels, very low-density lipoprotein cholesterol levels, low-density lipoprotein cholesterol levels, oxidative stress, levels of plasminogen activator inhibitor-1, von Willebrand factor levels and platelet aggregation and adhesion while improving diastolic function, vascular relaxation and tissue plasminogen activator activity [4]. Several observational analyses on patient populations with severe cardiovascular disease at baseline have shown cardioprotective benefits with metformin [22].

\section{Metabolic abnormalities associated with HIV}

In HIV infected patients Highly Active Antiretroviral Therapy (HAART) is used. This therapy is associated with metabolic 
abnormalities like that of glucose homeostasis including impaired glucose tolerance, hypertriglyceridemia, low HDL cholesterol and higher cardiovascular events. Protease inhibitors reduce insulin sensitivity and inhibit glucose transport mediated by Glucose Transporter (GLUT-4). Metformin therapy were used in these patients and found beneficial effects. Many studies of metformin and other insulin sensitizing agents used in HIV patients are currently underway with HAART linked with abnormal glucose metabolism and insulin resistance [5].

\section{Cancer}

Observational studies are being conducted in which metformin can be used in reducing the risk of cancer. Partly metformin causes the activation of AMPK. LKB1 a tumor suppressor is a regulator of AMPK, so increased activity of this pathway can exert anti-tumor effects $[23,24]$.

\section{Metformin Dose Adjustment}

Concurrently, the initiation of metformin therapy starts with lifestyle intervention at the time of diagnosis. In the absence of specific contraindications metformin is suggested as the initial pharmacological therapy, because of its effect on glycaemia, no weight gain or hypoglycaemia, high acceptance level and relatively less cost. Treatment with metformin as tolerated should be adjusted to its maximum effective dose over 1-2 months (Figure 2) [25].

\section{Adverse effects}

Metformin has been recommended as first-line therapy for T2DM due to its efficacy, safety (i.e., deficiency in weight gain and reduced hypoglycemia risk), comparatively reduced cost, and potential cardiovascular benefit [22]. Treatment with metformin is, nevertheless, very often associated with Gastrointestinal (GI) side effects in $20-30 \%$ of patients and the quality of life and treatment adherence is negatively affected in patients of T2DM. Metformin shows interference with the absorption of vitamin B12; however it is very rarely linked with anaemia [9]. The use of metformin is considered to be contraindicated in renal dysfunction because it has potential to increase the risk of lactic acidosis, which is extremely rare (less than one case per 100,000 treated patients) but is a fatal complication [25]. In $\sim 5 \%$ of patients severe GI symptoms develop, who discontinued the metformin treatment. This could deprive them of the advantageous effects of metformin. It is hypothesized that after oral administration of the drug GI intolerance is because of higher metformin concentration in the intestine [26]. The most common GI symptoms were diarrhea, heartburn, and nausea, followed by abdominal pain, bloating, and retching. Constipation was also reported in other study [27]. Flatulence, constipation, dyspepsia has also been seen [22]. Prevalence of GI symptoms is depicted in Table 1.

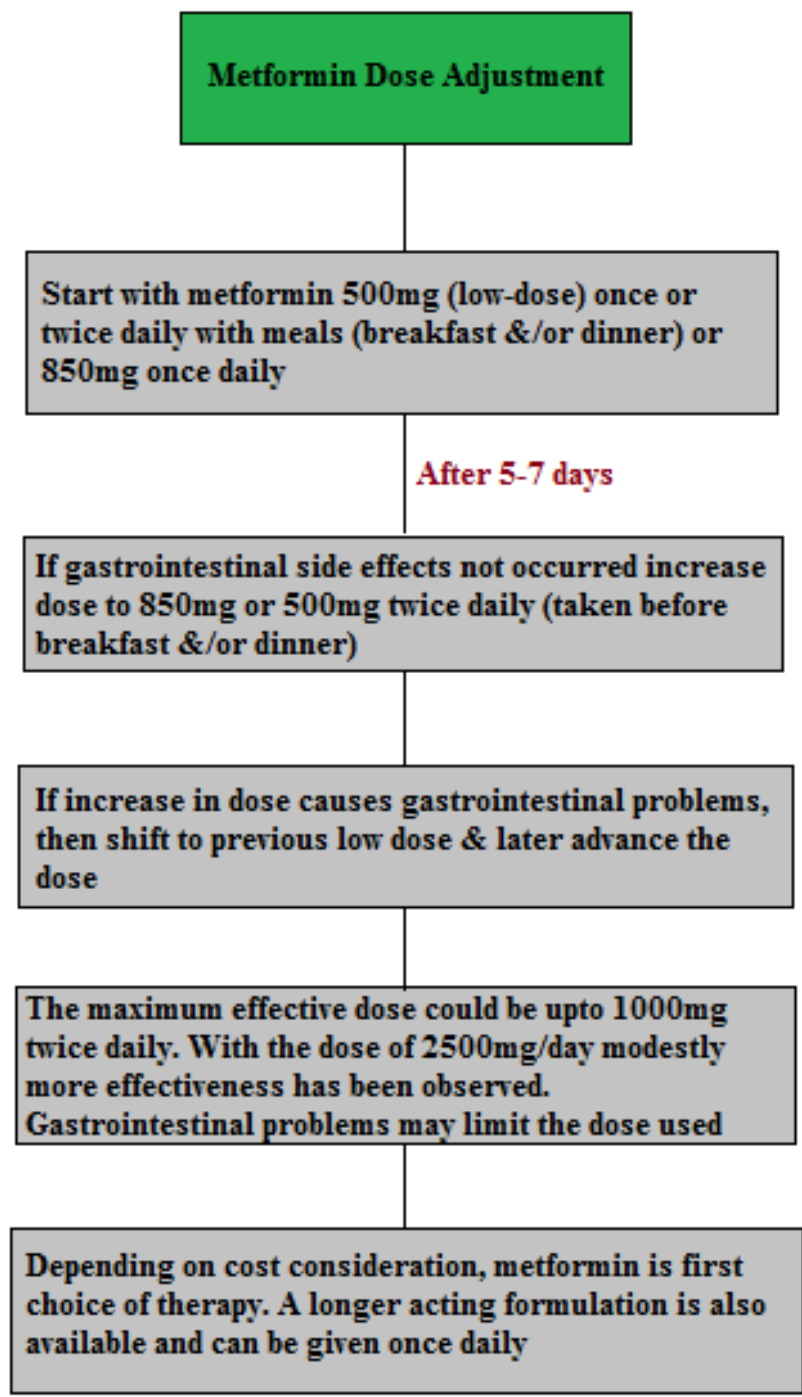

Figure 2. The dose adjustment of metformin.

Table 1. Prevalence of GI symptoms.

\begin{tabular}{ll}
\hline GI symptoms & Percentage \\
\hline Diarrhea & $62.1 \%$ \\
\hline Heartburn & $52.1 \%$ \\
\hline Nausea & $47.4 \%$ \\
\hline Abdominal pain & $35.5 \%$ \\
\hline Bloating & $35.2 \%$ \\
\hline Retching & $21.1 \%$ \\
\hline
\end{tabular}

\section{Mechanism of metformin induced gastrointestinal side effects}

The mechanism lying under the GI intolerance caused by metformin is unclear. However, there are different hypothesis proposed, including stimulation of intestinal secretion of serotonin, alteration in incretin and metabolism of glucose, and 
malabsorption of bile salts. It has been found that metformin structure has some similarities with 5-hydroxytrptamine 5-HT3 receptor selective agonists and, as mentioned earlier, is transported by Serotonin Reuptake Transporter (SERT). The release of serotonin (5-hydroxytrptamine (5-HT)) from the intestine results in the symptoms nausea, vomiting and diarrhea which are similar to those as linked with metformin intolerance. Therefore, one of the mechanisms lying under the metformin induced GI intolerance may relate to changes in serotonin transport or a direct metformin serotonergic-like effect. Duodenal biopsy study on metformin-naive individuals found that metformin stimulated the release of serotonin (5HT) from enterochromaffin cells [28]; however, this effect is not through the 5-HT3 receptor, as the response is not altered if receptor is inhibited. Alternatively it could be explained that, the uptake of metformin via SERT or Organic Cation Transporter (OCT1) results in decreased transport of serotonin and results in GI side-effects [29].

Within the intestine the bile acid pool is increased by metformin, mainly through decreased ileum absorption. This enterohepatic bile salts disruption has potential outcome for homeostasis of cholesterol, glucose homeostasis and enteroendocrine function. This could also account for metformin intolerance through changes in the microbiome and stool consistency. Furthermore, the alteration in the absorption of bile acid may cause an increase in the Glucagon-Like Peptide-1 (GLP-1) secretion, similarly observed with sequestrants of bile acid such as colesevelam [30]. The absorption of bile acid in the jejunum is a concentrationdependent, non-saturable and passive process; however ileal absorption is an active process [31]. It has also been proposed that a raised luminal concentration of bile salt would have an osmotic effect, which can cause diarrhoea linked with treatment of metformin [32].

Metformin is an organic cation, and its oral absorption is mediated by carrier proteins, hepatic uptake, and renal elimination. The membrane of enterocytes expresses many solute carrier transporters that are involved in the metformin absorption from the intestinal lumen. This includes Organic Cation Transporter (OCT) 1, Plasma Membrane Monoamine Transporter (PMAT), carnitine/cation transporter 1, and OCT3. The decreased metformin transport via OCT1 increases the metformin concentration in the intestine, resulting in higher risk of GI intolerance and discontinuation of drug. The underlying precise mechanism remains uncertain, suggesting that in enterocytes there is basolateral localization of OCT; If in the efflux of metformin OCT1 has a role (either by the basolateral or apical route), then inhibition could increase metformin concentration in the enterocytes. On the other hand, if in the transport of metformin from the lumen into the enterocytes OCT1 has a role, and then the inhibition of OCT1 could increase luminal concentration of metformin. Although proposed hypotheses of GI intolerance by metformin are inconclusive, and it is not clear whether adverse effects are due to the presence of drug in mucosa or in the lumen, raised concentration of metformin in the gut may affect intestinal concentration of serotonin, absorption of bile salts, or possibly may cause alteration in the microbiome. With reduced OCT1 transport an alternative mechanism for the higher metformin intolerance is that the inhibition of OCT1 is changing the systemic metformin concentrations, and it is the increased circulating concentration of metformin results in metformin intolerance [26]. Some of the actions of metformin causing GI intolerance are depicted in Figure 3.

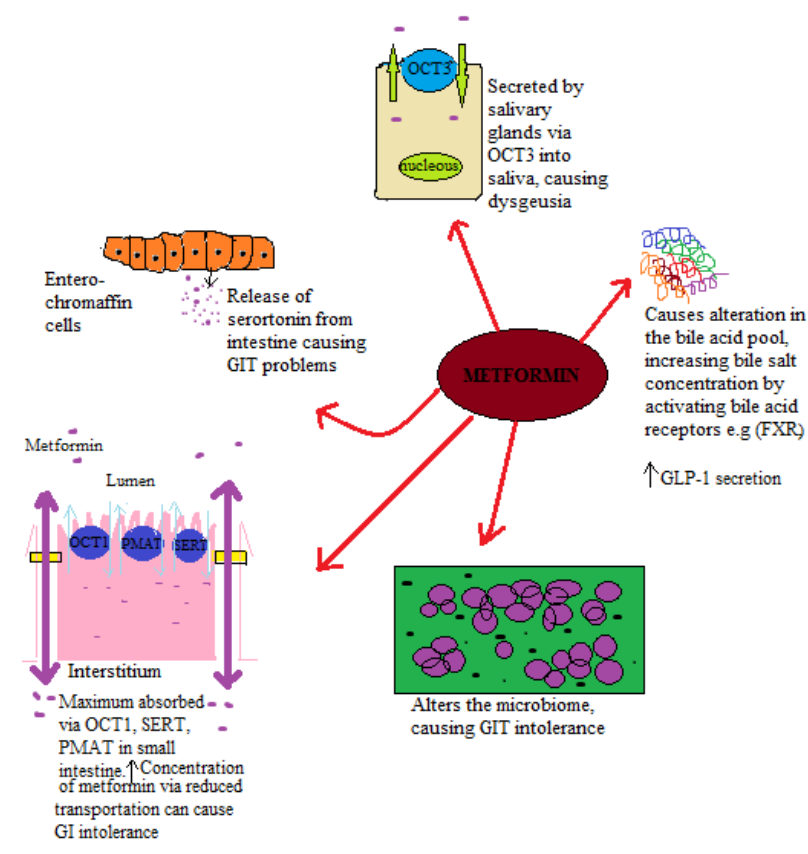

Figure 3. Some of the actions of metformin causing GI intolerance. The upward arrows show an increase: FXR: Farnesoid X Receptor; GLP-1: Glucagon-Like Peptide-1; OCT: Organic Cation Transporter; PMAT: Plasma Membrane Monoamine Transporter; SERT: Serotonin Transporter; GIT: Gastrointestinal Tract.

\section{References}

1. Bouchoucha M, Uzzan B, Cohen R. Metformin and digestive disorders. Diab Metabol 2011; 37: 90-96.

2. Kolata GB. The phenformin ban: is the drug an imminent hazard? Science 1979; 203: 1094-1096.

3. DeFronzo RA. Pharmacologic therapy for type 2 diabetes mellitus. Ann Int Med 1999; 131: 281-303.

4. Kirpichnikov D, McFarlane SI, Sowers JR. Metformin: an update. Ann Int Med 2002; 137: 25-33.

5. Hundal RS, Inzucchi SE. Metformin. Drugs 2003; 63: 1879-1894.

6. Tucker GT, Casey C, Phillips PJ, Connor H, Ward JD, Woods HF. Metformin kinetics in healthy subjects and in patients with diabetes mellitus. Br J Clin Pharmacol 1981; 12: 235-246.

7. Scheen AJ. Clinical pharmacokinetics of metformin. Clin Pharmacokinet 1996; 30: 359-371.

8. Robert F, Fendri S, Hary L, Lacroix C, Andrejak M, Lalau JD. Kinetics of plasma and erythrocyte metformin after acute administration in healthy subjects. Diab Metabol 2003; 29: 279-283. 
9. Bailey CJ, Turner RC. Metformin. N Engl J Med 1996; 334: 574-579.

10. DeFronzo RA, Goodman AM, Multicenter Metformin Study Group. Efficacy of metformin in patients with noninsulin-dependent diabetes mellitus. N Engl J Med 1995; 333: 541-549.

11. Harrigan RA, Nathan MS, Beattie P. Oral agents for the treatment of type 2 diabetes mellitus: pharmacology, toxicity, and treatment. ANN Emerg Med 2001; 38: 68-78.

12. Hundal RS, Krssak M, Dufour S, Laurent D, Lebon V, Chandramouli V, Shulman GI. Mechanism by which metformin reduces glucose production in type 2 diabetes. Diabetes 2000; 49: 2063-2069.

13. Rena G, Pearson ER, Sakamoto K. Molecular mechanism of action of metformin: old or new insights? Diabetologia 2013; 56: 1898-1906.

14. Zhou G, Myers R, Li Y, Chen Y, Shen X, Fenyk-Melody $\mathrm{J}$, Musi N. Role of AMP-activated protein kinase in mechanism of metformin action. J Clin Investig 2001; 108: 1167-1174.

15. Napolitano A, Miller S, Nicholls AW, Baker D, Van Horn S, Thomas E, Nunez DJ. Novel gut-based pharmacology of metformin in patients with type 2 diabetes mellitus. PLoS One 2014; 9: 100778.

16. Drucker DJ. The biology of incretin hormones. Cell Metabol 2006; 3: 153-165.

17. Stumvoll M, Nurjhan N, Perriello G, Dailey G, Gerich JE. Metabolic effects of metformin in non-insulin-dependent diabetes mellitus. N Engl J Med 1995; 333: 550-554.

18. Cusi K, Consoli A, Defronzo RA. Metabolic effects of metformin on glucose and lactate metabolism in noninsulin-dependent diabetes mellitus. J Clin Endocrinol Metabol 1996; 81: 4059-4067.

19. Christiansen MP, Linfoot PA, Neese RA, Hellerstein M. Metformin effects upon post absorptive intra hepatic carbohydrate fluxes. Sci Info Datab 1997.

20. Yu JG, Kruszynska YT, Mulford MI, Olefsky JM. A comparison of troglitazone and metformin on insulin requirements in euglycemic intensively insulin-treated type 2 diabetic patients. Diabetes 1999; 48: 2414-2421.

21. Wiernsperger NF, Bailey CJ. The antihyperglycaemic effect of metformin. Drugs 1999; 58: 31-39.

22. Scarpello JH, Howlett HC. Metformin therapy and clinical uses. Diab Vasc Dis Res 2008; 5: 157-167.

23. Shaw RJ, Lamia KA, Vasquez D, Koo SH, Bardeesy N, DePinho RA, Cantley LC. The kinase LKB1 mediates glucose homeostasis in liver and therapeutic effects of metformin. Science 2005; 310: 1642-1646.

24. Evans JM, Donnelly LA, Emslie-Smith AM, Alessi DR, Morris AD. Metformin and reduced risk of cancer in diabetic patients. BMJ 2005; 330: 1304-1305.

25. Nathan DM, Buse JB, Davidson MB, Heine RJ, Holman RR, Sherwin R, Zinman B. Management of hyperglycemia in type 2 diabetes: a consensus algorithm for the initiation and adjustment of therapy: a consensus statement from the American Diabetes Association and the European Association for the Study of Diabetes. Diab Care 2006; 29: 1963-1972.

26. Dujic T, Zhou K, Donnelly LA, Tavendale R, Palmer CN, Pearson ER. Association of organic cation transporter 1 with intolerance to metformin in type 2 diabetes: a GoDARTS study. Diabetes 2015; 64: 1786-1793.

27. Burton JH, Johnson M, Johnson J, Hsia DS, Greenway FL, Heiman ML. Addition of a gastrointestinal microbiome modulator to metformin improves metformin tolerance and fasting glucose levels. J Diab Sci Technol 2015; 9: 808-814.

28. Cubeddu LX, Bonisch H, Gothert M, Molderings G, Racke K, Ramadori G, Schworer H. Effects of metformin on intestinal 5-hydroxytryptamine (5-HT) release and on 5-HT 3 receptors. Naunyn-Schmiedebergs Arc Pharmacol 2000; 361: 85-91.

29. Yee SW, Lin L, Merski M, Keiser MJ, Gupta A, Zhang Y, Giacomini KM. Prediction and validation of enzyme and transporter off-targets for metformin. J Pharmacokinet Pharmacodynam 2015; 42: 463-475.

30. McCreight LJ, Bailey CJ, Pearson ER. Metformin and the gastrointestinal tract. Diabetologia 2016; 59: 426-435.

31. Carter D, Howlett HCS, Wiernsperger NF, Bailey CJ. Differential effects of metformin on bile salt absorption from the jejunum and ileum. Diab Obes Metabol 2003; 5: 120-125.

32. Scarpello JHB, Hodgson E, Howlett HCS. Effect of metformin on bile salt circulation and intestinal motility in type 2 diabetes mellitus. Diab Med 1998; 15: 651-656.

\section{*Correspondence to}

Saleha Sadeeqa

Institute of Pharmacy

Lahore College for Women University

Pakistan 